\title{
Electron bounce-cyclotron resonance in capacitive discharges at low magnetic fields
}

\author{
Sanket Patil, ${ }^{1}$ Sarveshwar Sharma $\odot,{ }^{1,2, *}$ Sudip Sengupta $\odot,{ }^{1,2}$ Abhijit Sen $\odot,{ }^{1,2}$ and Igor Kaganovich $\odot^{3}$ \\ ${ }^{1}$ Institute for Plasma Research, Bhat, Gandhinagar 382428 , India \\ ${ }^{2}$ Homi Bhabha National Institute, Anushaktinagar, Mumbai 400 094, India \\ ${ }^{3}$ Princeton Plasma Physics Laboratory, Princeton University, Princeton, New Jersey 08543, USA
}

(Received 5 December 2020; revised 9 November 2021; accepted 4 January 2022; published 28 January 2022)

\begin{abstract}
We report the existence of an enhanced operating regime for a high-frequency, low-pressure capacitively coupled plasma (CCP) discharge in the presence of a weak magnetic field applied parallel to the electrodes. Our particle-in-cell simulations show that the discharge operates at significantly higher plasma density and ion flux when the electron-cyclotron frequency equals half the applied RF frequency at a given voltage. The physical mechanism responsible for this behavior is a resonance between the oscillatory motion of the sheath edge and the electron bounce in the cyclotron motion, which is half of the cyclotron period. Hence we call this resonance the electron bounce cyclotron resonance. In each collision with the sheath the electrons gain a substantial amount of energy eventually sufficient to produce higher ionization near the sheath leading to increase in the ion flux. The effect is observed at a relatively weak magnetic field, about $10 \mathrm{G}$ at $60 \mathrm{MHz}$. The proposed effect can be used for enhancing the operational performance of CCP devices in industrial applications.
\end{abstract}

DOI: 10.1103/PhysRevResearch.4.013059

\section{INTRODUCTION}

Capacitively coupled plasma (CCP) discharges are widely used in industry for plasma processing applications, e.g., etching and deposition. The processing rates and the quality of the processed wafer depend on the ion flux incident on the substrate located at one of the electrodes and the energy of those ions. A discharge that yields a higher and more uniform ion flux with low ion energies could be more desirable for these applications. Several past studies carried out for singlefrequency CCP discharges have shown that it is difficult to have independent control of the ion flux and ion energy [1-9]. Alternate approaches to overcome this limitation have been explored in the form of dual-frequency CCPs [10-17], electrical asymmetric effects [18-22], and nonsinusoidal, tailored voltage wave form [23-31]. Applying a magnetic field parallel to the electrodes has also been found to enhance the performance of a CCP [32-36]. Although stronger magnetic fields (typically hundreds of Gauss) yield better performance, they can cause nonuniformity in the plasma and damage the wafer [37-40].

In this article, we report a significant enhancement in the performance of low-pressure CCP discharges at much lower magnetic-field strengths $(\sim 10 \mathrm{G})$. Simulations carried out over a range of magnetic-field strengths for a $60 \mathrm{MHz}$, 5 mTorr discharge reveal that the plasma density and the

\footnotetext{
*sarvesh@ipr.res.in.

Published by the American Physical Society under the terms of the Creative Commons Attribution 4.0 International license. Further distribution of this work must maintain attribution to the author(s) and the published article's title, journal citation, and DOI.
}

ion flux are greatly enhanced at a particular strength of the magnetic field (10.7 G). Recent findings in Ref. [41] also provide preliminary experimental evidence of this effect in a discharge operated at $40 \mathrm{MHz}$. The enhancement in performance is caused by resonance between the oscillatory motion of the sheath edge and the motion of electrons bouncing off the sheath edge, subsequently gyrating, and returning to the sheath after half a cyclotron period. Therefore, the resonance occurs when the electron cyclotron frequency $\left(f_{\mathrm{ce}}=\right.$ $\frac{e B}{m_{e}}$, where B, e, and $m_{e}$ are the external magnetic field, electronic charge, and mass, respectively) is equal to half the applied RF frequency $\left(f_{r f}\right)$. This resonance condition corresponds to quite low strengths of the magnetic field $(\sim 10$ G) for typical frequencies used for CCPs $(13.56-300 \mathrm{MHz})$. We call this resonance the bounce-cyclotron resonance because the main condition for the resonance is that half of the cyclotron period is equal to the RF period, which is different than the usual electron-cyclotron resonance (ECR) condition where the full cyclotron period is equal to the electric field period. The bounce-cyclotron resonance leads to a more effective energy gain by the electrons compared to that in the absence of a magnetic field, leading to a greater ionization of the neutral background gas and a resultant increase in the ion flux. This effect of a weak magnetic field on the discharge is distinct from the effects of much higher magnetic fields on the discharge [32,34-36]. In this article, we discuss the simulation results, the resonance mechanism, and the conditions for the existence of such a resonance.

\section{SIMULATION TECHNIQUE AND PARAMETERS}

Our simulations of a plasma bounded by two parallel electrodes have been carried out with the well-tested and widely used 1D-3V electrostatic direct implicit particle- 

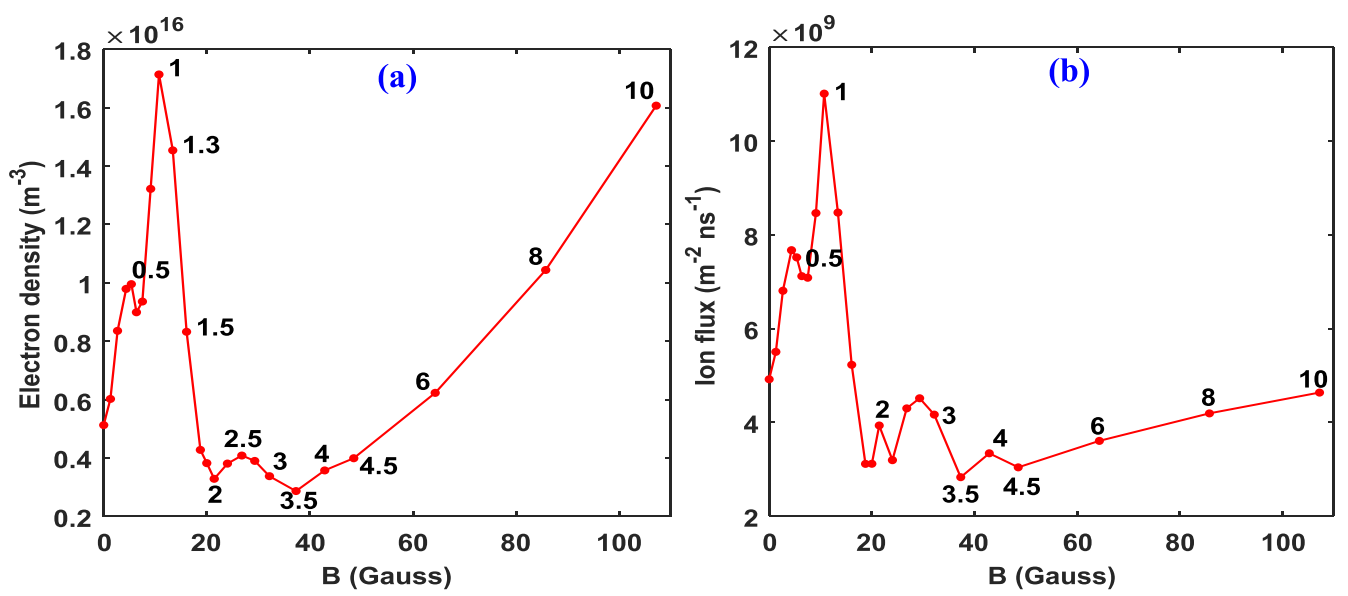

FIG. 1. (a) Dependence of the maximum plasma density in the discharge on the applied magnetic field; (b) dependence of the ion flux at the GE on the applied magnetic field. The numbers shown next to the curves indicate the $r$ value corresponding to the magnetic field.

in-cell (EDIPIC) code [42-47]. The code is based on the well-established particle-in-cell/Monte Carlo collision (PIC-MCC) method [48,49]. The types of electron-neutral collisions taken into account are elastic, excitation, and ionization collisions. For ions, ion-neutral elastic and charge exchange collisions have been considered. The metastable reactions are not important at low pressures and therefore have not been taken into account. The cross-sectional data used for the collisions have been taken from well-regarded sources [50,51]. The code evolves the positions and velocities of electrons and singly ionized ions $\mathrm{Ar}^{+}$. Note that although the code is $1 \mathrm{D}$ in position space, it is $3 \mathrm{D}$ in velocity space. Therefore it accurately simulates the 3D velocity of the charged particles, including the $E \times B$ effects in direction parallel to electrodes but does not include any polarization effects in directions other than normal to the wall. The neutral gas dynamics and secondary electron emission are not taken into account because their effects on the discharge properties are small at the low pressures considered here [52]. The neutral gas used in this study is argon at 5 mTorr pressure. The frequency and amplitude of the applied voltage are fixed at $60 \mathrm{MHz}$ and $100 \mathrm{~V}$, respectively. A harmonic voltage has been applied between the grounded electrode (GE) and the powered electrode (PE), which are $32 \mathrm{~mm}$ apart. Perfectly absorbing boundary conditions have been used, i.e., all charged particles get absorbed when they impinge on the electrodes. The initial electron and ion temperatures have been taken to be $2 \mathrm{eV}$ and $0.026 \mathrm{eV}(300 \mathrm{~K})$, respectively. The initial density is $5 \times 10^{15} \mathrm{~m}^{-3}$. The cell size $(\Delta x)$ used is $1 / 8$ times the initial Debye length $\left(\lambda_{D e}\right)$. The time step $(\Delta t)$ is calculated as $\Delta x /$ (maximum expected velocity), where maximum expected velocity is four times the initial thermal velocity of electrons. The time step thus satisfies the stability criterion $\omega_{p e} \Delta t<0.2$, where $\omega_{p e}$ is electron plasma frequency. This time step sufficiently resolves the cyclotron motion even at the highest magnetic field used $(107 \mathrm{G})$. The number of macro particles per cell is initially 400, giving the total number of particles to be $\sim 5 \times 10^{5}$. The external magnetic field $(B)$, applied parallel to the electrodes, has been quantified by the ratio $r=\frac{2 f_{\mathrm{ce}}}{f_{r f}}$ so that at resonance $r=1$. Here $r$ has been varied from 0 to 10 to capture the effect of a broad range of the magnetic-field values, which correspondingly varies from $0 \mathrm{G}$ to $107 \mathrm{G}$.

\section{RESULTS AND DISCUSSION}

Figure 1 shows the dependence of the maximum plasma density in the discharge and of the ion flux on the strength of the magnetic field. The numbers shown next to the curves indicate the $r$ value that corresponds to the magnetic-field strength. The simulation results show that the plasma density and the ion flux at the electrodes exhibit significant maximum at specific strengths of the magnetic field, i.e., specific values of $r=(0.5,1)$. As evident from Figure 1(a), there is a significant peak in the plasma density $\left(1.7 \times 10^{16} \mathrm{~m}^{-3}\right)$ at $r=1$ $(B=10.7 \mathrm{G})$ after which density rapidly decreases to increase again monotonically above $r=3.5(B=37.3 \mathrm{G})$ and reaches $1.6 \times 10^{16} \mathrm{~m}^{-3}$ at $r=10(B=107.2 \mathrm{G})$. The monotonic increase beyond $r=3.5$ has been already described in previous studies on magnetically enhanced CCPs [32,34-36,42]. The novel result is the sharp peak at $r=1$ whose origin we will discuss in this article. The dependence of the ion flux $\left(\Gamma_{i}=n_{i} u_{i}\right)$ at the electrodes on the magnetic field, as observed in Fig. 1(b), follows a trend similar to that of the density with a corresponding peak at $r=1$. The ion flux, however, does not increase as much as the density above $r=3.5$. This means that at a given amplitude of the applied voltage, the ion flux obtained at $r=1$ is significantly larger than that in the absence of magnetic field as well as for $r>3.5$; large magnetic fields $(r \sim 10)$ can produce high discharge densities but not proportionately high ion flux at the electrodes.

Figure 2(a) shows the variation of the average energy of the ions incident on the GE with the magnetic field. The average ion energy at $r=1(54.5 \mathrm{eV})$ is slightly lower than that in the absence of the magnetic field $(57.0 \mathrm{eV})$. This change can be ascribed to the decrease in the sheath width. Summarizing observations at $r=1$, a significantly higher ion flux with a slightly lower average ion energy is obtained at the electrodes compared with the discharge without an applied magnetic field.

We now discuss the physical origin of this effect. The trajectories of three electrons during multiple interactions 

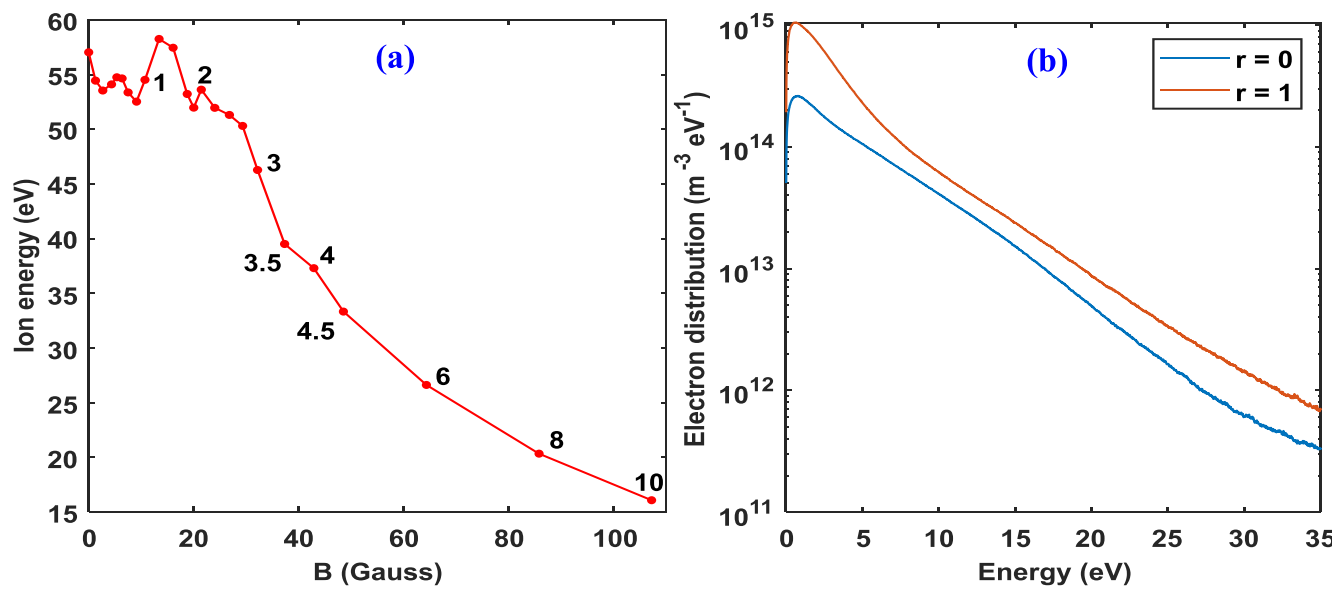

FIG. 2. (a) Variation of average energy of the ions incident on the GE with applied magnetic field. (b) Comparison of EEDFs as measured near the sheath edges in the absence and presence of the magnetic field.

with the sheath, as observed in the simulations, are shown in Fig. 3 for $\mathrm{B}=0 \mathrm{G}, \mathrm{B}=5.3 \mathrm{G}$ (corresponding to $r=0.5$ ), and $\mathrm{B}=10.7 \mathrm{G}$ (corresponding to $r=1$ ). In each case, electrons moving toward an electrode are reflected back into the bulk due to the strong electric field in the sheath. In a nearly collisionless discharge, these reflected electrons rarely collide with a neutral atom in the bulk. In the $r=0$ case, they reach the opposite sheath. The travel time between interactions with the two sheaths is different for electrons with different velocities [Fig. 3(a)]. As a result, subsequent interactions with the sheaths are not in the same phase of the sheath motion and are not generally resonant. Note that the electron motion for the $r=1$ case closely resembles pure cyclotron motion [Fig. 3(b)], since the RF electric fields are weak in the plasma $\left(<10^{3} \mathrm{~V} / \mathrm{m}\right)$. As a result, all electrons reflected by the left sheath return to the same sheath after half a cyclotron period irrespective of their velocities, since the cyclotron period does not depend on velocity for electrons with no velocity parallel to the electrodes at the time of collision with the sheath. We call this duration between subsequent reflections, or "bounces," the bounce time. For $r=1$, the bounce time is equal to the RF period. Therefore electrons reflected by a sheath at the expanding phase of an RF cycle will return to the sheath at the same phase and thereby undergo another energy gain from the interaction with the RF sheath. This process leads to a very effective generation of energetic electrons for the $r=1$ case as compared with the $r=0$ case. These electrons gain significant energy and contribute significantly to ionization near the sheath and increase ion flux. The electrons continue being in such a resonance until they collide with a neutral atom, which only happens once every few RF periods on average at these low pressures. This is evident in Fig. 2 which shows the electron energy distribution function (EEDF) near the sheath edges for the two cases as shown in Fig. 2(b). The number densities of electrons with energies above the ionization threshold $(15.76 \mathrm{eV})$ at the sheath
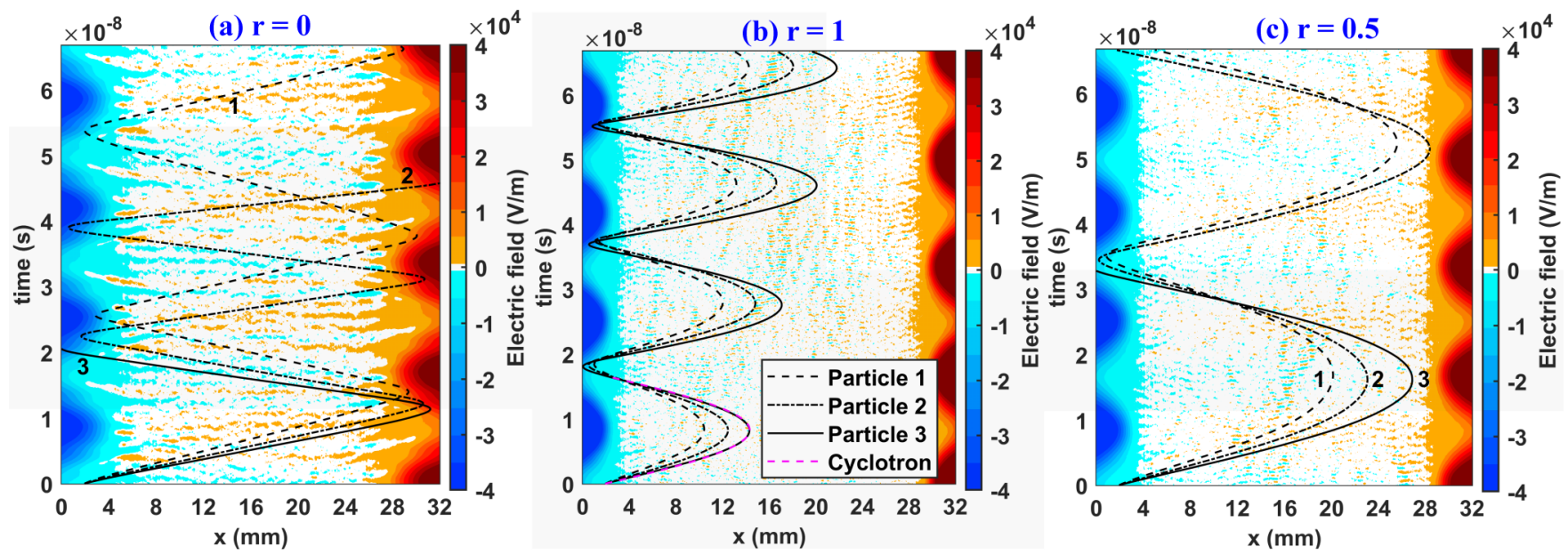

FIG. 3. Trajectories of reflected electrons having three different energies plotted onto the plot of electric field as a function of space coordinate $x$ and time. Particles 1, 2, and 3 have an initial energy of 4, 8, and $12 \mathrm{eV}$, respectively. It can be seen that for $r=0$, the three particles reach the opposite sheath at different times whereas for $r=0.5$ and $r=1$, the three particles return to the same sheath simultaneously. For $r=1$, the dotted magenta line shows pure cyclotron motion (no RF fields) of a particle with energy $12 \mathrm{eV}$. It can be seen that the motion of particle 3 closely resembles cyclotron motion. 
edges are $5.5 \times 10^{13} \mathrm{~m}^{-3}$ (for $r=0$ ) and $9.8 \times 10^{13} \mathrm{~m}^{-3}$ (for $r=1$ ). This enhanced density of energetic electrons leads to higher ionization of the neutrals and is the cause of the enhanced density observed for $r=1$.

Note that a similar enhancement in density and ion flux is also observed for $r=0.5$. However, the resonance is less effective in this case for two reasons. First, the reflected electrons return to the sheath after two RF periods, making it more likely (compared with $r=1$ ) for them to undergo collisions in the bulk plasma. Second, at such a low magnetic field, a significant number of electrons have a Larmor radius $\left(r_{L}=\right.$ $m_{e} v_{\perp} / e B$ where $v_{\perp}$ is the component of velocity perpendicular to the direction of B) large enough to reach the opposite sheath. Interaction with the opposite sheath can change the electron trajectory and hence eliminate the resonance.

This brings us to the first of the two conditions necessary for effective bounce-cyclotron resonance: The electron gyroradius must be smaller than the discharge gap for most electrons so that there are no interactions with the opposite sheath, which eliminate resonance by changing the electron trajectories. In the current study, for $r=1$, the population of electrons with energies above $35 \mathrm{eV}$, i.e., electrons with Larmor radii above $18.6 \mathrm{~mm}$, is practically nonexistent. Because this Larmor radius is less than the discharge gap of $26 \mathrm{~mm}$ [Fig. 3(b)], the resonance condition is satisfied. However, the magnetic field is weaker for $r=0.5$, and electrons with energies above $17 \mathrm{eV}$ have Larmor radii larger than the discharge gap. As a result, most ionizing electrons interact with the opposite sheath, and the resonance is ineffective. As another example, consider a discharge operating at a frequency of $13.56 \mathrm{MHz}$ and a discharge gap of $26 \mathrm{~mm}$. For $r=$ $1, \mathrm{~B}=2.4 \mathrm{G}$. In such a weak magnetic field, electrons with energies above $3.5 \mathrm{eV}$ would have Larmor radii larger than the discharge gap. Therefore, a significant fraction of electrons would reach the opposite sheath after reflection, which will eliminate the resonance condition. Because the electron Larmor radius is inversely proportional to the strength of the resonant magnetic field, which in turn is proportional to the applied RF frequency for resonance conditions, the requirement that the Larmor radius is less than the gap puts a lower limit on the RF frequency at a given discharge gap. This lower limit is expected to be in the range $10-40 \mathrm{MHz}$ for typical interelectrode gaps $(2-10 \mathrm{~cm})$ used in applications. Therefore, high-frequency (HF) discharges, which have received significant attention in recent years [53-63], are well-suited for enhancement due to the bounce-cyclotron resonance. The use of HF may produce some nonuniformities due to electromagnetic (EM) effects [64-73]. However, efforts to mitigate these effects have recently had some success [74,75], and it is an active area of research. Moreover, the use of a weak magnetic field can avoid the strong nonuniformity typically observed in conventional discharges that use much stronger magnetic fields [37-40]. Therefore the use of bounce-cyclotron resonance has a potential to be used in applications for HF RF discharges.

The second condition for effective resonance is that the discharge must be nearly collisionless. If electrons collide with neutrals in the bulk between consecutive bounces, their trajectories will be altered, and the resonance will be lost. Therefore the fraction of electrons bouncing off the sheath that contribute to the resonant effect is equal to the average probability that an electron does not collide with a neutral between consecutive bounces. This can be calculated as $P=\exp \left(-f_{\text {en }} T_{\text {bounce }}\right)=\exp \left(-f_{\text {en }} / f_{\text {rf }}\right)$, where $T_{\text {bounce }}=1 / f_{\text {rf }}$ is the bounce time. The resonance is ineffective if $P<<1$, i.e., if the ratio $f_{\text {en }} / f_{\text {rf }}$ is large, or equivalently, if the electron mean-free path is smaller than the electron gyroradius. In our simulations, $P=0.21$ at 5 mTorr, i.e., on average, $21 \%$ of the electrons bouncing off the sheath return for a consecutive sheath interaction. At 10 mTorr, where this fraction drops to $4 \%$, simulations reveal that the resonant effect is insignificant. Another example is Ref. [35], where a $13.56 \mathrm{MHz}, 10 \mathrm{mTorr}$ discharge was used, but no notable enhancement at $r=1$ was observed. Summarizing, there is an upper limit on the neutral gas pressure where the resonance effect is pronounced at a given applied RF frequency.

\section{CONCLUSION}

In conclusion, resonance is observed between the oscillatory motion of the sheath edge and the motion of electrons bouncing off the sheath edge. This resonance allows the electrons to effectively gain energy from the RF sheaths and cause higher ionization, resulting in higher ion flux impinging on the electrodes. This bounce-cyclotron resonance is characterized by the condition $r=1\left(f_{\mathrm{ce}}=\frac{f_{\mathrm{rf}}}{2}\right)$, which corresponds to relatively weak magnetic-field strengths. The resonance is expected to be particularly effective for low-pressure, high-frequency CCP discharges. Our theoretical findings are supported by preliminary experiments [41] and could provide provide an operational regime for enhanced performance of CCP discharges in industrial applications.

\section{ACKNOWLEDGMENTS}

A.S. thanks the Indian National Science Academy (INSA) for their support under the INSA Senior Scientist Fellowship scheme. The work of I.K. was supported by the Princeton Collaborative Research Facility (PCRF), which is funded by the U.S. Department of Energy (DOE) under Contract No. DE-AC02-09CH11466.
[1] I. D. Kaganovich, O. V. Polomarov, and C. E. Theodosiou, Revisiting the anomalous RF field penetration into a warm plasma, IEEE Trans. Plasma Sci. 34, 696 (2006).

[2] E. Kawamura, M. A. Lieberman, and A. J. Lichtenberg, Stochastic heating in single and dual frequency capacitive discharges, Phys. Plasmas 13, 053506 (2006).
[3] M. A. Lieberman, Analytical solution for capacitive RF sheath, IEEE Trans. Plasma Sci. 16, 638 (1988).

[4] V. A. Godyak, Steady-state low pressure rf discharge, Sov. J. Plasma Phys. 2, 78 (1976).

[5] O. A. Popov and V. A. Godyak, Power dissipated in lowpressure radio-frequency plasma, J. Appl. Phys. 57, 53 (1985). 
[6] I. D. Kaganovich, Anomalous Capacitive Sheath with Deep Radio-Frequency Electric-Field Penetration, Phys. Rev. Lett. 89, 265006 (2002).

[7] S. Sharma, S. K. Mishra, and P. K. Kaw, Observation of transient electric fields in particle-in-cell simulation of capacitively coupled discharges, Phys. Plasmas 21, 073511 (2014).

[8] S. Sharma and M. M. Turner, Simulation study of stochastic heating in single-frequency capacitively coupled discharges with critical evaluation of analytical models, Plasma Sources Sci. Technol. 22, 035014 (2013).

[9] S. Sharma and M. M. Turner, Simulation study of wave phenomena from the sheath region in single frequency capacitively coupled plasma discharges; field reversals and ion reflection, Phys. Plasmas 20, 073507 (2013)

[10] H. H. Goto, H. D. Lowe, and T. Ohmi, Independent control of ion density and ion bombardment energy in a dual RF excitation plasma, IEEE Trans. Semicond. Manufact. 6, 58 (1993).

[11] J. Robiche, P. C. Boyle, M. M. Turner, and A. R. Ellingboe, Analytical model of a dual frequency capacitive sheath, J. Phys. D: Appl. Phys. 36, 1810 (2003).

[12] H. C. Kim, J. K. Lee, and J. W. Shon, Analytic model for a dual frequency capacitive discharge, Phys. Plasmas 10, 4545 (2003).

[13] M. M. Turner and P. Chabert, Collisionless Heating in Capacitive Discharges Enhanced by Dual-Frequency Excitation, Phys. Rev. Lett. 96, 205001 (2006).

[14] S. Sharma and M. M. Turner, Critical evaluation of analytical models for stochastic heating in dual-frequency capacitive discharges, J. Phys. D: Appl. Phys. 46, 285203 (2013).

[15] S. K. Karkari, A. R. Ellingboe, and C. Gaman, Direct measurement of spatial electron density oscillations in a dual frequency capacitive plasma, Appl. Phys. Lett. 93, 071501 (2008).

[16] P. C. Boyle, A. R. Ellingboe, and M. M. Turner, Independent control of ion current and ion impact energy onto electrodesin dual frequency plasma devices, J. Phys. D: Appl. Phys. 37, 697 (2004).

[17] S. Sharma and M. M. Turner, Investigation of wave emission phenomena in dual frequency capacitive discharges using particle-in-cell simulation, J. Phys. D: Appl. Phys. 47, 285201 (2014).

[18] U. Czarnetzki, J. Schulze, E. Schungel, and Z. Donko, The electrical asymmetry effect in capacitively coupled radio-frequency discharges, Plasma Sources Sci. Technol. 20, 024010 (2011).

[19] B. G. Heil, U. Czarnetzki, R. P. Brinkmann, and T. Mussenbrock, On the possibility of making a geometrically symmetric RF-CCP discharge electrically asymmetric, J. Phys. D: Appl. Phys. 41, 165202 (2008).

[20] B. Bruneau, T. Novikova, T. Lafleur, J. P. Booth, and E. V. Johnson, Ion flux asymmetry in radiofrequency capacitivelycoupled plasmas excited by sawtooth-like waveforms, Plasma Sources Sci. Technol. 23, 065010 (2014).

[21] B. Bruneau, T. Gans, D. O'Connell, A. Greb, E. Johnson, and J.-P. Booth, Strong Ionization Asymmetry in a Geometrically Symmetric Radio Frequency Capacitively Coupled Plasma Induced by Sawtooth Voltage Waveforms, Phys. Rev. Lett. 114, 125002 (2015).

[22] E. Schungel, I. Korolov, B. Bruneau, A. Derzsi, E. Johnson, D. O'Connell, T. Gans, J. P. Booth, Z. Donko, and J. Schulze, Tailored voltage waveform capacitively coupled plasmas in electronegative gases: Frequency dependence of asymmetry effects, J. Phys. D: Appl. Phys. 49, 265203 (2016).
[23] D. J. Economou, Tailored ion energy distributions on plasma electrodes, J. Vac. Sci. Technol. A 31, 050823 (2013).

[24] T. Lafleur, P. Chabert, M. M. Turner, and J. P. Booth, Theory for the self-bias formation in capacitively coupled plasmas excited by arbitrary waveforms, Plasma Sources Sci. Technol. 22, 065013 (2013).

[25] P. Diomede, D. J. Economou, T. Lafleur, J. P. Booth, and S. Longo, Radio-frequency capacitively coupled plasmas in hydrogen excited by tailored voltage waveforms: Comparison of simulations with experiments, Plasma Sources Sci. Technol. 23, 065049 (2014).

[26] A. Derzsi, T. Lafleur, J. P. Booth, I. Korolov, and Z. Donkó, Experimental and simulation study of a capacitively coupled oxygen discharge driven by tailored voltage waveforms, Plasma Sources Sci. Technol. 25, 015004 (2016).

[27] T. Lafleur, P. A. Delattre, E. V. Johnson, and J. P. Booth, Separate control of the ion flux and ion energy in capacitively coupled radio-frequency discharges using voltage waveform tailoring, Appl. Phys. Lett. 101, 124104 (2012).

[28] T. Lafleur, Tailored-waveform excitation of capacitively coupled plasmas and the electrical asymmetry effect, Plasma Sources Sci. Technol. 25, 013001 (2016).

[29] X. V. Qin, Y. H. Ting, and A. E. Wendt, Tailored ion energy distributions at an rf-biased plasma electrode, Plasma Sources Sci. Technol. 19, 065014 (2010).

[30] H. Shin, W. Zhu, L. Xu, V. M. Donnelly, and D. J. Economou, Control of ion energy distributions using a pulsed plasma with synchronous bias on a boundary electrode, Plasma Sources Sci. Technol. 20, 055001 (2011).

[31] S. Sharma, S. K. Mishra, P. K. Kaw, A. Das, N. Sirse, and M. M. Turner, Collisionless sheath heating in currentdriven capacitively coupled plasma discharges via higher order sinusoidal signals, Plasma Sources Sci. Technol. 24, 025037 (2015).

[32] M. A. Lieberman, A. J. Lichtenberg, and S. E. Savas, Model of magnetically enhanced, capacitive RF discharges, IEEE Trans. Plasma Sci. 19, 189 (1991).

[33] D. Cheng, D. Maydan, S. Somekh, K. R. Stalder, D. L. Andrews, M. Chang, J. M. White, J. Y. Wong, V. J. Zeitlin, and D. N. Wang, U.S. Patent No. 5, 215, 619 (1993).

[34] D. A. W. Hutchinson, M. M. Turner, R. A. Doyle, and M. B. Hopkins, The effects of a small transverse magnetic field upon a capacitively coupled RF discharge, IEEE Trans. Plasma Sci. 23, 636 (1995).

[35] M. M. Turner, D. A. W. Hutchinson, R. A. Doyle, and M. B. Hopkins, Heating Mode Transition Induced by a Magnetic Field in a Capacitive rf Discharge, Phys. Rev. Lett. 76, 2069 (1996).

[36] J.-C. Park and B. Kang, Reactor modeling of magnetically enhanced capacitive RF discharge, IEEE Trans. Plasma Sci. 25, 499 (1997).

[37] M. J. Buie, J. T. P. Pender, and M. Dahimene, Characterization of the etch rate non-uniformity in a magnetically enhanced reactive ion etcher, J. Vac. Sci. Technol., A 16, 1464 (1998).

[38] M. J. Kushner, Modeling of magnetically enhanced capacitively coupled plasma sources: Ar discharges, J. Appl. Phys. 94, 1436 (2003).

[39] E. V. Barnat, P. A. Miller, and A. M. Paterson, Rf discharge under the influence of a transverse magnetic field, Plasma Sources Sci. Technol. 17, 045005 (2008).

[40] Y. Fan, Y. Zou, J. Sun, T. Stirner, and D. Wang, Study of the effects of a transverse magnetic field on radio frequency 
argon discharges by two-dimensional particle-in-cell-MonteCarlo collision simulations, Phys. Plasmas 20, 103507 (2013).

[41] Q. Z. Zhang, J. Y. Sun, W. Q. Lu, J. Schulze, Y. Q. Guo, and Y. N. Wang, Resonant sheath heating in weakly magnetized capacitively coupled plasmas due to electron-cyclotron motion, Phys. Rev. E 104, 045209 (2021).

[42] S. Sharma, I. D. Kaganovich, A. V. Khrabrov, P. Kaw, and A. Sen, Spatial symmetry breaking in single-frequency CCP discharge with transverse magnetic field, Phys. Plasmas 25, 080704 (2018).

[43] D. Sydorenko, Particle-in-cell simulations of electron dynamics in low pressure discharges with magnetic fields, Ph.D. thesis, University of Saskatchewan, Canada, 2006.

[44] M. D. Campanell, A. V. Khrabrov, and I. D. Kaganovich, Instability, collapse, and oscillation of sheaths caused by secondary electron emission, Phys. Plasmas 19, 123513 (2012).

[45] J. P. Sheehan, N. Hershkowitz, I. D. Kaganovich, H. Wang, Y. Raitses, E. V. Barnat, B. R. Weatherford, and D. Sydorenko, Kinetic Theory of Plasma Sheaths Surrounding Electron-Emitting Surfaces, Phys. Rev. Lett. 111, 075002 (2013).

[46] M. Campanell and H. Wang, Influence of emitted electrons transiting between surfaces on plasma-surface interaction, Appl. Phys. Lett. 103, 104104 (2013).

[47] J. Carlsson, A. Khrabrov, I. Kaganovich, T. Sommerer, and D. Keating, Validation and benchmarking of two particle-in-cell codes for a glow discharge, Plasma Sources Sci. Technol. 26, 014003 (2016).

[48] C. K. Birdsall, Plasma Physics via Computer Simulation (Adam Hilger, Bristol, 1991).

[49] R. W. Hockney and J. W. Eastwood, Computer Simulation Using Particles (Adam Hilger, Bristol, 1988).

[50] R. Shahid and M. J. Kushner, Argon metastable densities in radio frequency $\mathrm{Ar}, \mathrm{Ar} / \mathrm{O} 2$ and $\mathrm{Ar} / \mathrm{CF} 4$ electrical discharges, J. Appl. Phys. 82, 2805 (1997).

[51] L. Lauro-Taroni, M. M. Turner, and N. StJ. Braithwaite, Analysis of the excited argon atoms in the GEC RF reference cell by means of one-dimensional PIC simulations, J. Phys. D: Appl. Phys. 37, 2216 (2004).

[52] M. A. Lieberman and A. J. Lichtenberg, Principles of Plasma Discharges and Materials Processing (Wiley, NJ, 2005).

[53] S. Wilczek, J. Trieschmann, J. Schulze, E. Schuengel, R. P. Brinkmann, A. Derzsi, I. Korolov, Z. Donko, and T. Mussenbrock, The effect of the driving frequency on the confinement of beam electrons and plasma density in low-pressure capacitive discharges, Plasma Sources Sci. Technol. 24, 024002 (2015).

[54] S. Sharma, N. Sirse, P. K. Kaw, M. M. Turner, and A. R. Ellingboe, Effect of driving frequency on the electron energy distribution function and electron-sheath interaction in a low pressure capacitively coupled plasma, Phys. Plasmas 23, 110701 (2016).

[55] K. Bera, D. Hoffman, S. Shannon, G. Delgadino, and Y. Ye, Frequency optimization for capacitively coupled plasma source, IEEE Trans. Plasma Sci. 33, 382 (2005).

[56] S. Sharma, A. Sen, N. Sirse, M. M. Turner, and A. R. Ellingboe, Plasma density and ion energy control via driving frequency and applied voltage in a collisionless capacitively coupled plasma discharge, Phys. Plasmas 25, 080705 (2018).

[57] S. Sharma, N. Sirse, A. Sen, J. S. Wu, and M. M. Turner, Electric field filamentation and higher harmonic generation in very high frequency capacitive discharges, J. Phys. D: Appl. Phys. 52, 365201 (2019).

[58] P. A. Miller, E. V. Barnat, G. A. Hebner, P. A. Paterson, and J. P. Holland, Spatial and frequency dependence of plasma currents in a $300 \mathrm{~mm}$ capacitively coupled plasma reactor, Plasma Sources Sci. Technol. 15, 889 (2006).

[59] R. R. Upadhyay, I. Sawada, P. L. G. Ventzek, and L. L. Raja, Effect of electromagnetic waves and higher harmonics in capacitively coupled plasma phenomena, J. Phys. D: Appl. Phys. 46, 472001 (2013).

[60] S. Sharma, N. Sirse, M. M. Turner, and A. R. Ellingboe, Influence of excitation frequency on the metastable atoms and electron energy distribution function in a capacitively coupled argon discharge, Phys. Plasmas 25, 063501 (2018).

[61] S. Wilczek, J. Trieschmann, J. Schulze, Z. Donko, R. P. Brinkmann, and T. Mussenbrock, Disparity between current and voltage driven capacitively coupled radio frequency discharges, Plasma Sources Sci. Technol. 27, 125010 (2018).

[62] S. Sharma, N. Sirse, A. Sen, M. M. Turner, and A. R. Ellingboe, Influence of select discharge parameters on electric field transients triggered in collisionless very high frequency capacitive discharges, Phys. Plasmas 26, 103508 (2019).

[63] S. Sharma, N. Sirse, A. Kuley, and M. M. Turner, Electric field nonlinearity in very high frequency capacitive discharges at constant electron plasma frequency, Plasma Sources Sci. Technol. 29, 045003 (2020).

[64] P. Chabert and N. Braithwaite, Physics of Radio-Frequency Plasmas (Cambridge University Press, Cambridge, UK, 2011).

[65] A. Perret, P. Chabert, J. P. Booth, J. Jolly, J. Guillon, and P. Auvray, Ion flux nonuniformities in large-area high-frequency capacitive discharges, Appl. Phys. Lett. 83, 243 (2003).

[66] I. Sawada, P. L. G. Ventzek, B. Lane, T. Ohshita, R. R. Upadhyay, and L. L. Raja, Relationship between center-peaked plasma density profiles and harmonic electromagnetic waves in very high frequency capacitively coupled plasma reactors, Jpn. J. Appl. Phys. 53, 03DB01 (2014).

[67] D.-Q. Wen, E. Kawamura, M. A. Lieberman, A. J. Lichtenberg, and Y.-N. Wang, Nonlinear series resonance and standing waves in dual-frequency capacitive discharges, Plasma Sources Sci. Technol. 26, 015007 (2017).

[68] D.-Q. Wen, E. Kawamura, M. A. Lieberman, A. J. Lichtenberg, and Y.-N. Wang, A nonlinear electromagnetics model of an asymmetrically-driven, low pressure capacitive discharge, Phys. Plasmas 24, 083517 (2017).

[69] E. Kawamura, D.-Q. Wen, M. A. Lieberman, and A. J. Lichtenberg, Effect of a dielectric layer on plasma uniformity in high frequency electronegative capacitive discharges, J. Vac. Sci. Technol., A 35, 05C311 (2017).

[70] D.-Q. Wen, E. Kawamura, M. A. Lieberman, A. J. Lichtenberg, and Y.-N. Wang, Two-dimensional particle-in-cell simulations of standing waves and wave-induced hysteresis in asymmetric capacitive discharges, J. Phys. D: Appl. Phys. 50, 495201 (2017).

[71] K. Zhao, Y. X. Liu, E. Kawamura, D.-Q. Wen, M. A. Lieberman, and Y. N. Wang, Experimental investigation of standing wave effect in dual-frequency capacitively coupled argon discharges: role of a low-frequency source, Plasma Sources Sci. Technol. 27, 055017 (2018). 
[72] E. Kawamura, M. A. Lieberman, and A. J. Lichtenberg, Symmetry breaking in high frequency, symmetric capacitively coupled plasmas, Phys. Plasmas 25, 093517 (2018).

[73] K. Zhao, D. Q. Wen, Y. X. Liu, M. A. Lieberman, D. J. Economou, and Y. N. Wang, Observation of Nonlinear Standing Waves Excited by Plasma-Series-Resonance-Enhanced Harmonics in Capacitive Discharges, Phys. Rev. Lett. 122, 185002 (2019).
[74] K. Zhao, Z. X. Su, J. R. Liu, Y. X. Liu, Y. R. Zhang, J. Schulze, Y. H. Song, and Y. N. Wang, Suppression of nonlinear standing wave excitation via the electrical asymmetry effect, Plasma Sources Sci. Technol. 29, 124001 (2020).

[75] D. Han, Z. Su, K. Zhao, Y. Liu, F. Gao, and Y. Wang, Experimental investigation of the electromagnetic effect and improvement of the plasma radial uniformity in a large-area, very-high frequency capacitive argon discharge, Plasma Sci. Technol. 23, 055402 (2021). 